\title{
Exploring the Mechanism of Scutellaria baicalensis Georgi Efficacy against Oral Squamous Cell Carcinoma Based on Network Pharmacology and Molecular Docking Analysis
}

\author{
Fanfan Hou $\mathbb{D},{ }^{1}$ Yang Liu, ${ }^{1}$ YaHsin Cheng, ${ }^{2}$ Ni Zhang, ${ }^{1}$ Wenpeng Yan, ${ }^{1}$ and Fang Zhang $\mathbb{D}^{1}$ \\ ${ }^{1}$ Stomatology Hospital, Shanxi Medical University, Taiyuan 030001, China \\ ${ }^{2}$ Department of Physiology, School of Medicine, China Medical University, Taichung, Taiwan \\ Correspondence should be addressed to Fang Zhang; doctor-fangzh@hotmail.com
}

Received 1 March 2021; Accepted 26 May 2021; Published 13 July 2021

Academic Editor: Arham Shabbir

Copyright (c) 2021 Fanfan Hou et al. This is an open access article distributed under the Creative Commons Attribution License, which permits unrestricted use, distribution, and reproduction in any medium, provided the original work is properly cited.

Background. Scutellaria baicalensis Georgi (SBG) has been widely shown to induce apoptosis and inhibit invasion and migration of various cancer cells. Increased evidence shows that SBG may be useful to treat oral squamous cell carcinoma (OSCC). However, the biological activity and possible mechanisms of SBG in the treatment of OSCC have not been fully elucidated. This study aimed to clarify the bioactive component and multitarget mechanisms of SBG against OSCC using network pharmacology and molecular docking. Methods. Traditional Chinese Medicine Systems Pharmacology (TCMSP) database was used to predict the active components in SBG, and putative molecular targets of SBG were identified using the Swiss Target Prediction database. OSCCrelated targets were screened by GeneCards, Online Mendelian Inheritance in Man (OMIM), and Therapeutic Target Database (TTD). Then, we established protein-protein interaction (PPI), compound-target-disease (C-T-D), and compound-targetpathway (C-T-P) networks by Cytoscape to identify the main components, core targets, and pharmacological pathways of SBG against OSCC via applying data mining techniques and topological parameters. Metascape database was utilized for Gene Ontology (GO) and pathway enrichment analysis. The potential interaction of the main components with core targets was revealed by molecular docking simulation, and for the correlation between core targets and OSCC prognosis analysis, the Kaplan-Meier Plotter online database was used. Results. There were 25 active compounds in SBG and 86 genes targeted by OSCC. A total of 141 signaling pathways were identified, and it was found that the PI3K-Akt signaling pathway may occupy core status in the anti-OSCC system. GO analysis revealed that the primary biological processes were related to apoptosis, proliferation, and migration. Molecular docking results confirmed that core targets of OSCC had a high affinity with the main compounds of SBG. Conclusion. Our study demonstrated multicomponent, multitarget, and multipathway characteristics of SBG in the treatment of OSCC and provided a foundation for further drug development research.

\section{Introduction}

Oral squamous cell carcinoma (OSCC) is one of the most ubiquitous head and neck cancers and is prevalent worldwide, with about 350,000 newly diagnosed patients in 2018 [1]. Tobacco use, human papillomavirus (HPV), and alcohol consumption are considered the independent risk factors for OSCC [2]. Currently, OSCC requires a combination of treatments, including surgery, chemotherapy, radiotherapy, and immunotherapy [3]. Despite numerous attempts with different chemotherapy regimens and molecular target-based therapies, the overall 5-year survival rate of OSCC remains at $50 \%-60 \%$. However, chemotherapy for patients who cannot undergo surgical resection may prolong survival by about $15 \%$ while leading to the undesirable side effects of chemotherapy and drug resistance $[3,4]$. Therefore, there is a great need for more effective therapeutic drugs to treat OSCC.

Scutellaria baicalensis Georgi (SBG) belongs to the Lamiaceae herb family with extensive biological and pharmacological activities, whose desiccative radix is an important component of the fundamental herb used in Traditional Chinese medicine (TCM) [5]. The active ingredients in SBG 
have important pharmacological effects including antioxidant, antibacterial, antitumor, and anti-inflammatory $[5,6]$. Recently, SBG has been used to treat many diseases such as cancers, inflammation, hypertension, and immunologic disorders [7]. A wealth of evidence indicates that SBG may serve as essential source of novel therapeutic candidates for the treatment of OSCC due to its low side effects and remarkable activities [8-10]. However, the pharmacological mechanism of SBG in treating OSCC has not been fully elucidated for SBG is an herb containing various components.

TCM including SBG has the characteristics of "multicomponent, multitarget, and multipathway system.” [11] The recent development of network pharmacology has allowed the researcher to gain an understanding of the relationship of drugs with diseases and biological systems from a "Compound-Target-Disease" perspective [12]. Furthermore, molecular docking can predict the affinity of ligand and explain the mechanism of ligand action from the molecular level via exploring the interaction between ligand and receptor [13]. Kaplan-Meier Plotter online database was used to analyze the correlation between the core targets and the prognosis of OSCC further to verify the reliability of network pharmacology [14]. Utilizing these complementary techniques, we explored SBG targets and key signaling pathways that may provide potential strategies for treating OSCC. The flow diagram of the study is shown in Figure 1.

\section{Materials and Methods}

2.1. Acquisition of the Effective Components and Target of SBG. Traditional Chinese Medicine Systems Pharmacology (TCMSP, version: 2.3, https://tcmspw.com/tcmsp.php) database was used to collect the effective components of SBG [15]. Pharmacokinetic information retrieval filters absorption, distribution, metabolism, and excretion (ADME) were used to retrieve bioactive compounds for further analysis; we set oral bioavailability (OB) $\geq 30 \%$ and drug-likeness (DL) $\geq 0.18$ as screening criteria [16]. After integrating with the results obtained from the above databases, the $2 \mathrm{D}$ molecular structures of potential active chemical constituents of SBG were downloaded from the PubChem (https://pubchem. ncbi.nlm.nih.gov/) [17] and were input into Swiss Target Prediction database (http://www.swisstargetprediction.ch/) [18]; click "Homo sapiens" to view all putative targets of the chemical constituents. The results are stored in TSV format for the following analysis.

2.2. Fishing for Putative Targets of OSCC. OSCC-related target genes were mined using GeneCards (https://www. genecards.org Version 5.0.0) [19], Online Mendelian Inheritance in Man (OMIM, https://omim.org/, updated March 21, 2020) [20], and Therapeutic Target Database (TTD, http://db.idrblab.org/ttd/, updated June 1, 2020) [21] using "oral squamous cell carcinoma" as the keywords.

2.3. Obtaining Common Targets for the Disease and Drug. To collect candidate targets for OSCC treatment, the presumed targets of SBG were mapped to OSCC-related targets to obtain common targets. The screened chemical targets and disease targets are imported into the Draw Venn Diagram (http://bioinformatics.psb.ugent.be/webtools/Venn/) platform for analysis [22], and then the common targets of both the disease and the drug obtained are used as the core targets for subsequent analysis.

2.4. Drawing the Component-Target-Disease Network. Cytoscape (https://www.cytoscape.org/, version:3.7.2) was used to visualize the component-target-disease network to reflect the complex links among active constituents, potential targets, and diseases [23]. The active components of SBG and common therapeutic targets were imported into Cytoscape software, and the main components were identified according to the topological parameters.

2.5. Protein-Protein Interaction (PPI) Network Map of Common Targets and Core Targets'Screening. Search Tool for the Retrieval of Interacting Genes/Proteins (STRING, http:// stringdb.org/, version: 11.0) database was used to derive the PPI network [24]. Species was set as "Homo sapiens," and the confidence score was set at the highest level $(\geq 0.900)$; other parameters were set at default. Then, core targets were screened according to topological analysis. The file in TSV format was exported from the STRING database, and the downloaded results were uploaded to Cytoscape software for analysis.

2.6. Gene Ontology (GO) Functional Enrichment and Kyoto Encyclopedia of Genes and Genomes (KEGG) Pathway Analysis. Further analysis is required to reveal the specific mechanism of SBG in OSCC treatment. The common targets were uploaded into the Metascape system (http://metascape. org/, updated September 16, 2020) to complete GO functional analysis and KEGG pathways analysis [25], limited to "Homo sapiens," and significance levels were set to $P<0.01$.

2.7. Construction of the Component-Target-Pathway Network. Based on common targets of compound-disease and top predicted pathways, a "component-target-pathway" regulatory network was constructed using Cytoscape software [26].

2.8. Analysis of Core Target Genes and Overall Survival. The Kaplan-Meier Online website (http://kmplot.com/ analysis/) and both the Cancer Genome Atlas (TCGA) and Gene Expression Omnibus (GEO) databases were used to analyze the relationship between the expression level of core targets and cancer prognosis [14, 27]. We used data from the Pan-cancer of the Kaplan-Meier Online website for "head-neck squamous cell carcinoma" to analyze the relationship between core target genes and OSCC patient prognosis. A total of 499 patients with OSCC were identified, and $\log$-rank $P<0.05$ was statistically significant. 


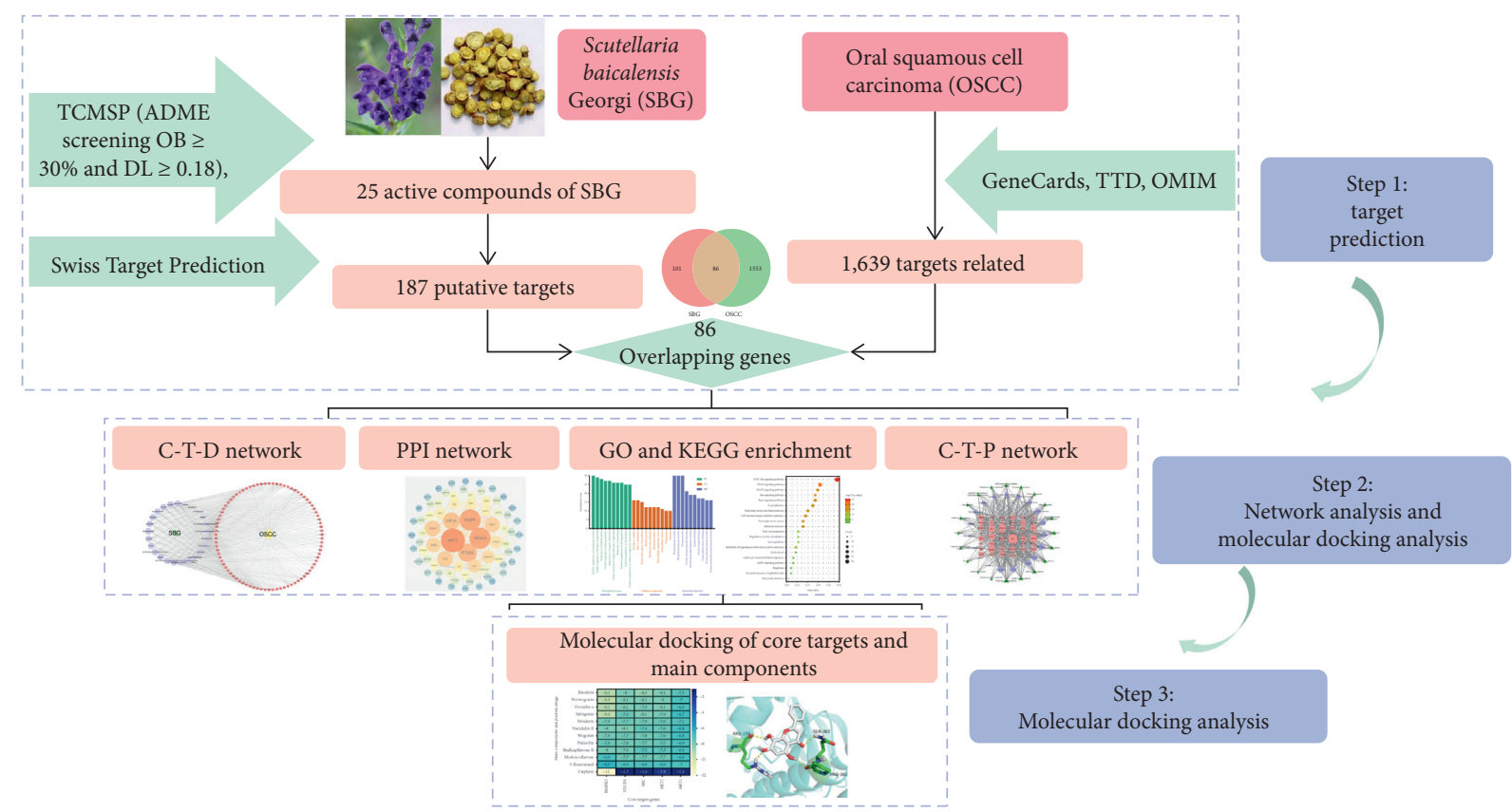

Figure 1: A flow diagram based on a cohesive integration strategy of network pharmacology and molecular docking.

2.9. Molecular Docking Analyses of the Main Active Components and Core Targets. A molecular docking tool (Systems Dock) was used to determine if the main components of SBG have the binding capacity with core targets. We selected core targets in the PPI and Component-TargetsPathways networks as potential receptors based on the degree value. The PubChem database was used to obtain 3D structures of the main active components of SBG, and the crystal structures of core target genes were directly obtained from the RCSB PDB database (https://www.rcsb. org/) [26]. AutoDock Tools 1.5.6 software was used to process the above protein receptors and ligands routinely and save them as a PDBQT file. Auto grid module was used to obtain the active docking site, run the program for molecular docking, and receive relevant binding energy (affinity). Autodock Vina 1.1.2 was used to perform bioinformatics studies and molecular docking. PyMOL 1.7.x software-based visualization analyses showed the conformation with the best affinity. The main active components of SBG were compared with 5-fluorouracil and cisplatin, which are the first-line treatment of OSCC [4]. Finally, a heat map was made based on the strongest affinity of core targets and main ingredients of SBG.

\section{Results}

3.1. Potential Targets of SBG. A total of 25 active chemical ingredients of SBG were obtained through the screening criteria properties ( $\mathrm{OB} \geq 30 \%$ and $\mathrm{DL} \geq 0.18$ ) based on TCMSP (Table 1 and Supplementary File, Table S1). The 2D SDF structure of these 25 compounds was uploaded to search for the potential therapeutic targets from the Swiss Target Prediction database, and 2,102 component-related targets were predicted (Supplementary File, Table S2). In total, there were 187 potential targets of SBG after duplicates were removed.

3.2. Potential Targets of OSCC. GeneCards, OMIM, and TTD were used to collect OSCC-related targets. By using the keywords "oral squamous cell carcinoma" in sequence, 4,586 OSCC-related targets were obtained from GeneCards, and then we chose 1,147 targets two times greater than the median of relevance score from GeneCards, 469 OSCCrelated genes from OMIM, and 1 OSCC-related target from TTD, respectively (Supplementary File, Table S3). As a result, a total of 1,639 targets associated with OSCC pathogenesis were obtained after duplicates had been deleted.

3.3. Converting Putative Targets of SBG against OSCC into Network and Analysis. Based on the above results, we identified 86 common targets of SBG into pathological targets by the intersection of 187 putative targets of SBG and 1,639 OSCC-related targets. The 86 common targets served as the putative therapeutic targets of SBG against OSCC (Figure 2(a), Supplementary File, Table S4). Furthermore, 86 potential genes were uploaded to the STRING database for analysis. There were a total of 86 nodes, 239 edges, and 5.56 average node degrees. Further analysis was done using the Cytoscape software, and the PPI network was visualized (Figure 2(b), Supplementary File, Table S5). The top 20 core targets were then selected (Figure 2(c)). From the PPI network and the bar graph (Figure 2(c)), we found that the leading core targets are phosphoinositide-3-kinase regulatory subunit 1 (PIK3R1), SRC proto-oncogene (SRC), AKT serine/ threonine kinase 1 (AKT1), mitogen-activated protein kinase 3 (MAPK3), and vascular endothelial growth factor 
TABLE 1: Basic information of 25 potential active components in SBG.

\begin{tabular}{|c|c|c|c|c|c|}
\hline Number & Mol ID & Molecule name & $\mathrm{OB}(\%)$ & DL & Molecular structure \\
\hline 1 & MOL000173 & Wogonin & 30.68 & 0.23 & \\
\hline 2 & MOL000552 & 5,2'-Dihydroxy-6,7,8-trimethoxyflavone & 31.71 & 0.35 & \\
\hline 3 & MOL002714 & Baicalein & 33.52 & 0.21 & \\
\hline 4 & MOL002909 & 5,7,2,5-Tetrahydroxy-8,6-dimethoxyflavone & 33.82 & 0.45 & \\
\hline 5 & MOL001689 & Acacetin & 34.97 & 0.24 & \\
\hline 6 & MOL012246 & 5,7,4'-Trihydroxy-8-methoxyflavanone & 74.24 & 0.26 & \\
\hline 7 & MOL012245 & 5,7,4'-Trihydroxy-6-methoxyflavanone & 36.63 & 0.27 & \\
\hline 8 & MOL002908 & 5,8,2'-Trihydroxy-7-methoxyflavone & 37.01 & 0.27 & \\
\hline 9 & MOL002925 & $5,7,2^{\prime}, 6^{\prime}$-Tetrahydroxyflavone & 37.01 & 0.24 & \\
\hline 10 & MOL012266 & Rivularin & 37.94 & 0.37 & \\
\hline 11 & MOL002926 & Dihydrooroxylin A & 38.72 & 0.23 & \\
\hline 12 & MOL000525 & Norwogonin & 39.4 & 0.21 & \\
\hline 13 & MOL002913 & Dihydrobaicalin_qt & 40.04 & 0.21 & \\
\hline 14 & MOL002910 & Carthamidin & 41.15 & 0.24 & \\
\hline 15 & MOL002914 & Eriodyctiol (flavanone) & 41.35 & 0.24 & \\
\hline 16 & MOL002928 & Oroxylin-A & 41.37 & 0.23 & \\
\hline
\end{tabular}


TABLE 1: Continued.

\begin{tabular}{|c|c|c|c|c|c|}
\hline Number & Mol ID & Molecule name & OB (\%) & DL & Molecular structure \\
\hline 17 & MOL008206 & Moslosooflavone & 44.09 & 0.25 & \\
\hline 18 & MOL002917 & Viscidulin II & 45.05 & 0.33 & \\
\hline 19 & MOL002915 & Salvigenin & 49.07 & 0.33 & \\
\hline 20 & MOL000228 & Alpinetin & 55.23 & 0.20 & \\
\hline 21 & MOL002927 & Skullcapflavone II & 69.51 & 0.44 & \\
\hline 22 & MOL002932 & Panicolin & 76.26 & 0.29 & \\
\hline 23 & MOL001458 & Coptisine & 30.67 & 0.86 & \\
\hline 24 & MOL002897 & Epiberberine & 43.09 & 0.78 & \\
\hline 25 & MOL002937 & Dihydrooroxylin & 66.06 & 0.23 & \\
\hline
\end{tabular}

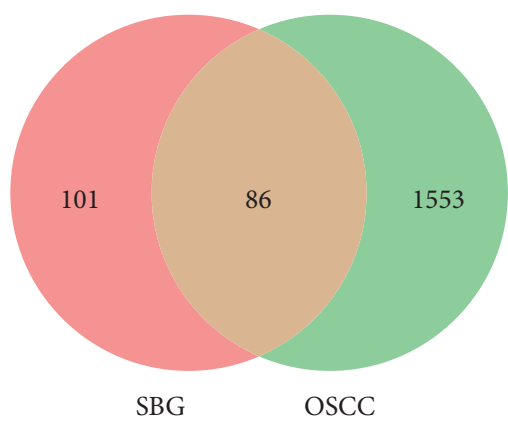

(a)

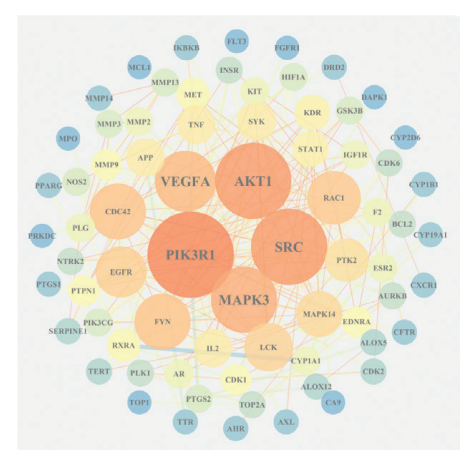

(b)

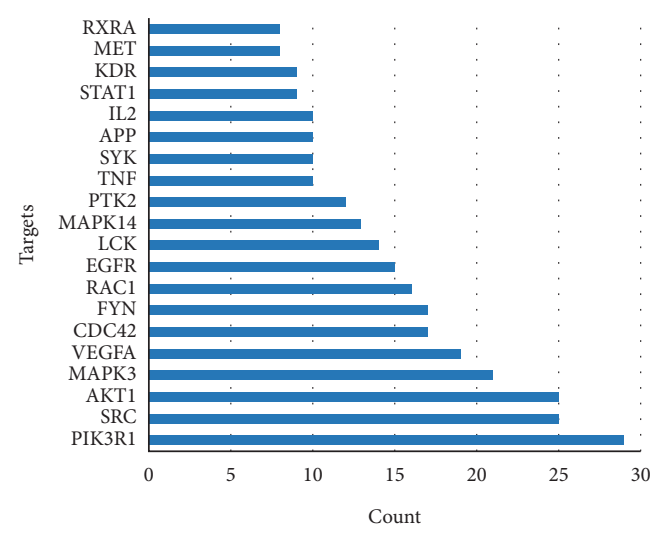

(c)

FIgURE 2: Construction of the PPI network and identification of the core targets. (a) Venn diagram of the intersection of 187 targets of SBG and 1,639 targets of OSCC. (b) PPI network of potential core targets related to SBG and OSCC. The nodes in lighter color and a more significant size represented a higher degree. The edges in lighter color and a wider size presented a higher combined score. (c) The top 30 core targets in the PPI network (the $y$-axis shows the top 20 significant targets, and the $x$-axis shows the count of interconnected targets).

A (VEGFA) (Table 2); these were considered as the core targets of SBG and may play a pivotal role in the development of OSCC.
3.4. Visualizing the Compound-Target-Disease Network. To build a compound-target-disease network diagram, we imported the 25 active ingredients of SBG and 86 common 
TABLE 2: Basic information about the 5 core targets.

\begin{tabular}{lccccc}
\hline Number & Uniport ID & Protein name & Gene name & Degree & Closeness \\
\hline 1 & P27986 & Phosphoinositide-3-kinase regulatory subunit 1 & PIK3R1 & 29 & 0.51079137 \\
2 & P12930 & SRC proto-oncogene & SRC & 25 & 0.50714286 \\
3 & P31750 & AKT serine/threonine kinase 1 & AKT1 & 25 & 0.51079137 \\
4 & P27361 & Mitogen-activated protein kinase 3 & MAPK3 & 21 & 0.48965517 \\
5 & P15692 & Vascular endothelial growth factor A & VEGFA & 19 & 0.5035461 \\
\hline
\end{tabular}

targets into the Cytoscape software resulting in a total of 139 nodes and 546 connections in this network (Figure 3). According to topological analysis (Supplementary File, Table S6), we found that the leading hub compounds are baicalein, norwogonin, wogonin, oroxylin-A, salvigenin, moslosooflavone, rivularin, skullcapflavone II, and viscidulin II, which are also the core components in SBG with anti-OSCC effect.

3.5. GO and KEGG Pathway Enrichment Analysis. We imported the 86 potential therapeutic targets into the Metascape system for GO and KEGG analysis to further elucidate the mechanism of SBG against OSCC. Biological processes (BP), molecular functions (MF), and cellular components (CC) related to the GO results revealed the functions of these potential targets. A total of 1,846 significant enriched GO entries were obtained from Metascape, including 1,653 BP, $115 \mathrm{MF}$, and $78 \mathrm{CC}$ entries based on $P<0.01$ (Supplementary File, Table S7). Out of 1,653 BP GO terms, the most enriched BP terms were positive regulation of transferase activity, cellular response to nitrogen compounds, and response to oxidative stress (Figure 4(a)). And out of $115 \mathrm{MF}$ GO terms, the most important terms associated SBG treatment were protein kinase activity, phosphotransferase activity, and kinase activity (Figure 4(a)). Among 78 CC GO terms associated SBG treatment, receptor complex, side of membrane, and perinuclear region of the cytoplasm were included in the top 10 entries (Figure 4(a)). A total of 141 pathways were enriched based on $P<0.01$ (Supplementary File, Table S8); the pathways with the highest enrichment level included PI3K-Akt, EGFR, and MAPK signaling, primarily involved in the immune-related and inflammatory-related function. Based on $P$ values and counts of hit genes, the top $10 \mathrm{GO}$ entries, and top 20 KEGG pathways were chosen (Figures 4(a) and 4(b)).

3.6. The Compound-Target-Pathway Network of SBG for OSCC. The compound-target-pathway network analysis is shown in Figure 5, and the specific data are given in Table 3. The network relationship between the top 20 pathways and their regulated target genes is shown in the diagram. According to topological analysis, PIK3R1, SRC, AKT1, MAPK3, and VEGFA had the highest volume in genes and thus may be critical in the SBG treatment of OSCC. A relatively high volume consistent on the compound-targetdisease network was also observed among baicalein, norwogonin, wogonin, oroxylin-A, and salvigenin.
3.7. Analysis of the Core Target Genes on Median Survival of OSCC. We used the Kaplan-Meier Plotter online database to analyze the correlation between five identified genes (PIK3R1, SRC, AKT1, MAPK3, and VEGFA) and OSCC prognosis. Correlation analysis showed that the expressions of PIK3R1, SRC, AKT1, MAPK3, and VEGFA were all related to the median survival time of OSCC patients $(P<0.05)$. The median survival time of the patients with high expression of PIK3R1 and MAPK3 was better than that of the low expression group, and increased expression of SRC, AKT1, and VEGFA was worse than low SRC, AKT1, and VEGFA expression as shown in Figure 6. The results suggest that PIK3R1, SRC, AKT1, MAPK3, and VEGFA as core target genes of SBG were significant associations with survival time in patients with OSCC.

3.8. Molecular Docking Analysis. Molecular docking analysis provided a visual interaction between the chemical components of SBG and OSCC-related genes. Cisplatin and 5-fluorouracil are used as positive drugs for molecular docking. The smaller the binding energy, the stronger the binding force between the component and target. Affinity $<-4.25 \mathrm{kcal} / \mathrm{mol}$ means that ligands and receptors have the possibility of combination, affinity $<-5.00 \mathrm{kcal} / \mathrm{mol}$ indicates good binding strength, and affinity $<-7.00 \mathrm{kcal} / \mathrm{mol}$ suggests satisfactory binding strength [28]. As previously described, 60 pairs resulted from molecular docking analysis (Supplementary File, Table S9). From the above results, as compared to 5-fluorouracil and cisplatin, the main components and core targets have better binding activity (affinity $<-7.00 \mathrm{kcal} / \mathrm{mol}$ ). Baicalein has four hydrogen bonds with the amino acid residues bound to VEGFA and formed a stable complex (Figure 7(a)). The specific molecular docking results are shown in Table 4, and a visual result is shown in Figure 7. We placed the best pairs of compounds-targets into PyMOL software for visualization, and the specific protein-ligand interactions of docking are shown in Figure 8.

\section{Discussion}

OSCC is an inadequately treated cancer that affects the quality of life of patients [29]. Surgery is primarily used for early treatment, and combination therapy (chemotherapy and radiotherapy) is commonly used to prevent recurrence and improve efficacy, but there are side effects. Therefore, it is essential to develop new drugs to reduce the morbidity and mortality of OSCC. TCM has been widely accepted as a mainstream form of complementary and alternative therapy 


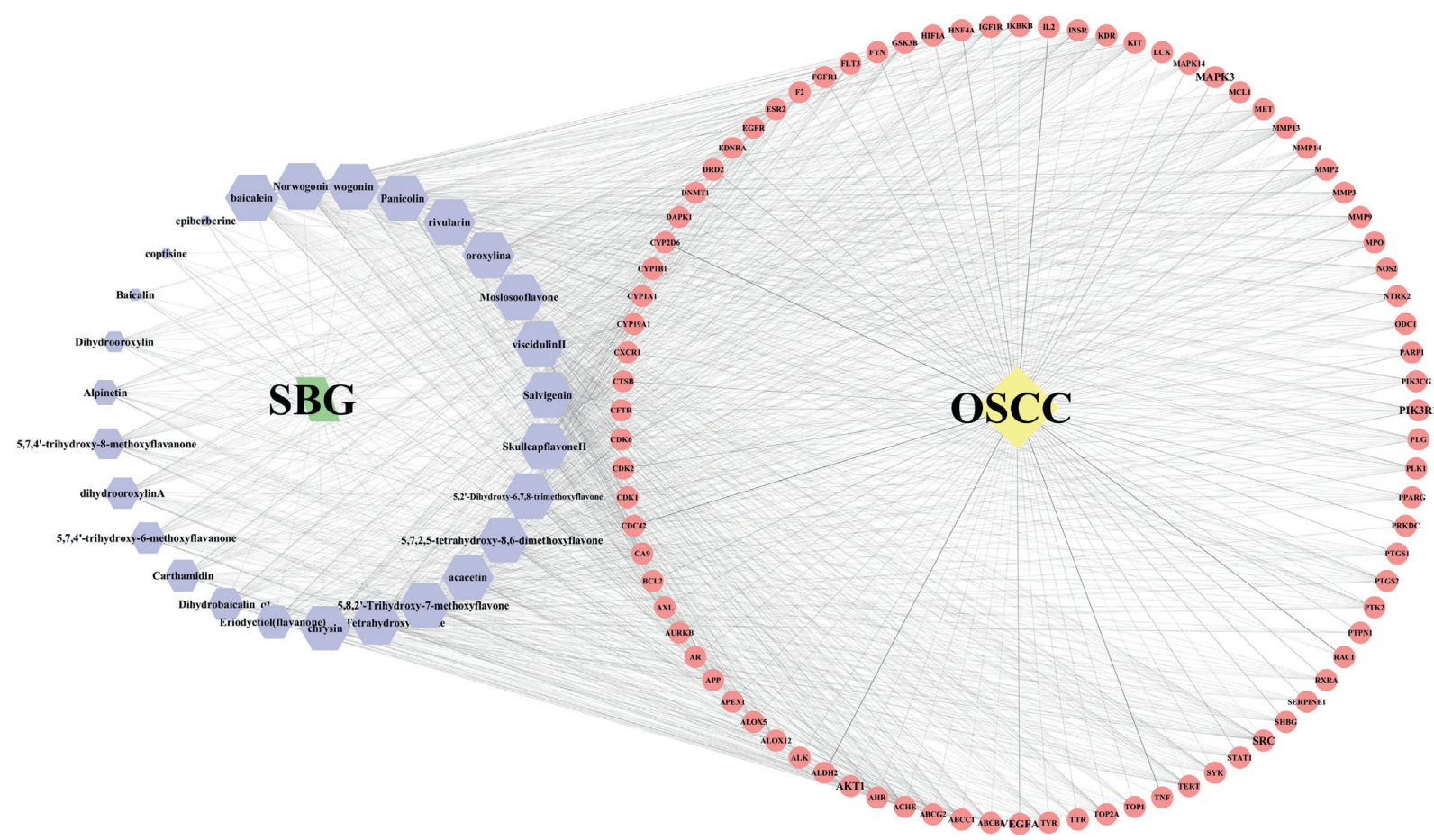

FIGURE 3: Construction of the compound-target-disease network (the blue hexagon nodes represent active compounds of SBG, and pink ellipse nodes represent potential therapeutic targets. Nodes' sizes are proportional to their degree).

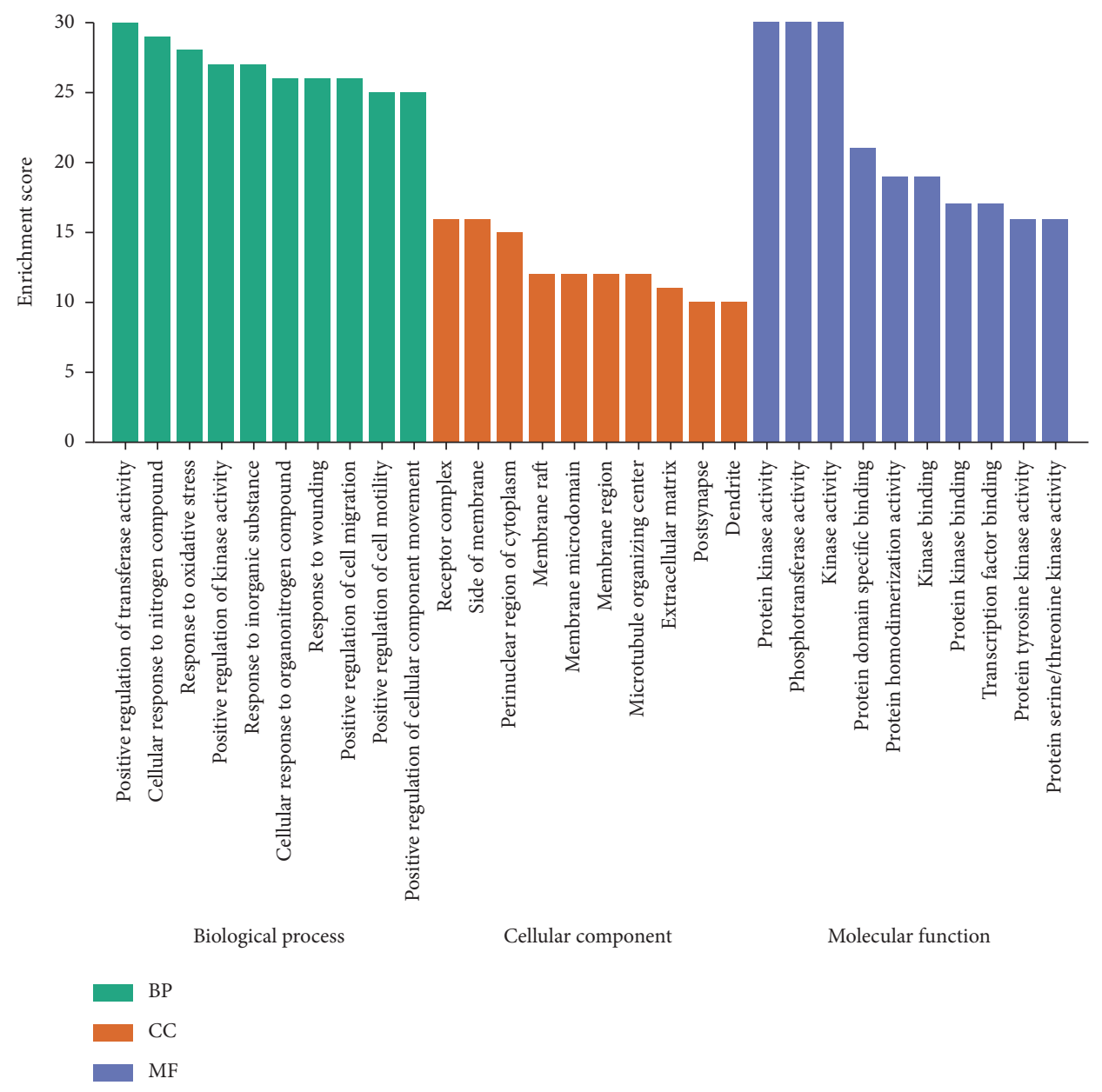

(a) 


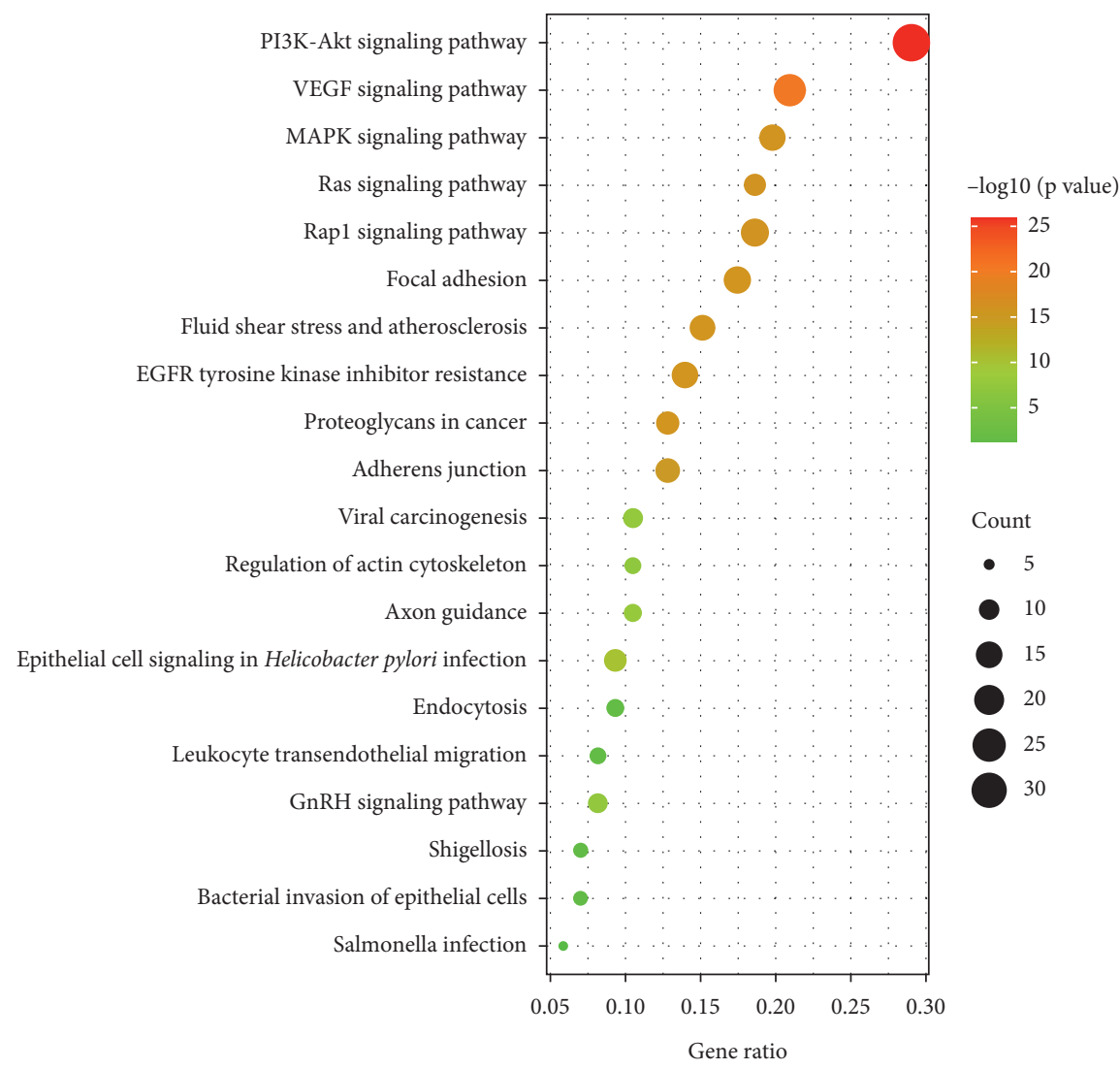

(b)

FIGURE 4: GO enrichment and KEGG pathway enrichment analysis of the 86 potential therapeutic targets. (a) GO enrichment analysis: the top 10 biological processes, molecular functions, and cell components, $P<0.01$. (b) Bubble chart of the top 20 KEGG pathways, $P<0.01$.

beneficial to cancer patients because it inhibits and kills tumor cells and can reduce radiotherapy and chemotherapy's adverse effects [11]. TCM treatment also improves patients' quality of life and immunity, reduces clinical symptoms, and prolongs survival time [30]. SBG is one of the TCM that has been reported to possess anticancer, antioxidant, anti-inflammatory, antiviral, and antibacterial activities [31].

Our data showed 25 active compounds in SBG that act on 86 different targets associated with OSCC. Five flavonoid compounds, baicalein, wogonin, oroxylin-A, salvigenin, and norwogonin, are highly connected potential therapeutic targets and pathways of SBG against OSCC which can be defined as decisive compounds in OSCC according to the topological parameters. The five compounds play protective roles against tumor cell development, and dietary intake of flavonoids can significantly reduce the risk of cancer and inflammation [32]. The previous study has shown that baicalein can induce cell cycle arrest and apoptosis to treat and prevent cancer without adverse side effects [33]. Gao et al. have demonstrated that baicalein suppresses OSCC cells' growth through Sp1/NF- $\kappa$ B dependent mechanism [8]. Wogonin has been thoroughly studied for its anticancer, antiviral, and antioxidant activity [34]. Wogonin's tumor inhibitory properties may be associated with targeting PI3KAkt and MAPK pathways, inhibiting the cell cycle arrest and overcoming drug resistance [35]. Oroxylin-A possesses pharmacological effects of anticancer, anti-inflammatory, neuroprotective, and anticoagulant activities [36]. Slavigenin is also a potent anticancer agent active against various human cancer cell lines [37], and it has been found to have antiproliferative, anti-inflammatory, and cytotoxic effects [38]. Hypoxia induces EMT and increases migration and metastasis in OSCC $[39,40]$. And previous studies have articulated that norwogonin can attenuate hypoxia-induced oxidative stress and apoptosis [41]. Therefore, these main components of SBG play an essential role in anti-OSCC, which is consistent with previous studies.

By integrating network analyses, we revealed that SBG acts on multiple targets using multiple signaling pathways, mainly PIK3R1, SRC, AKT1, MAPK3, and VEGFA, potentially core target genes that could be pivotal in the development of OSCC. PIK3R1 is the predominant regulatory isoform of PI3K, a tumor-suppressor gene in OSCC, regulating cancer cell proliferation $[42,43]$. The expression and activity of SRC are associated with advanced malignancy and poor prognosis in a variety of human cancers. SRC plays a vital role in cell proliferation, invasion, movement, and signal transduction [44]. AKT1 plays a key oncogene role in human OSCC cells and may be a critical target for novel therapy development [45]. As a member of the mitogenactivated protein kinase (MAPK) family, the abnormal 


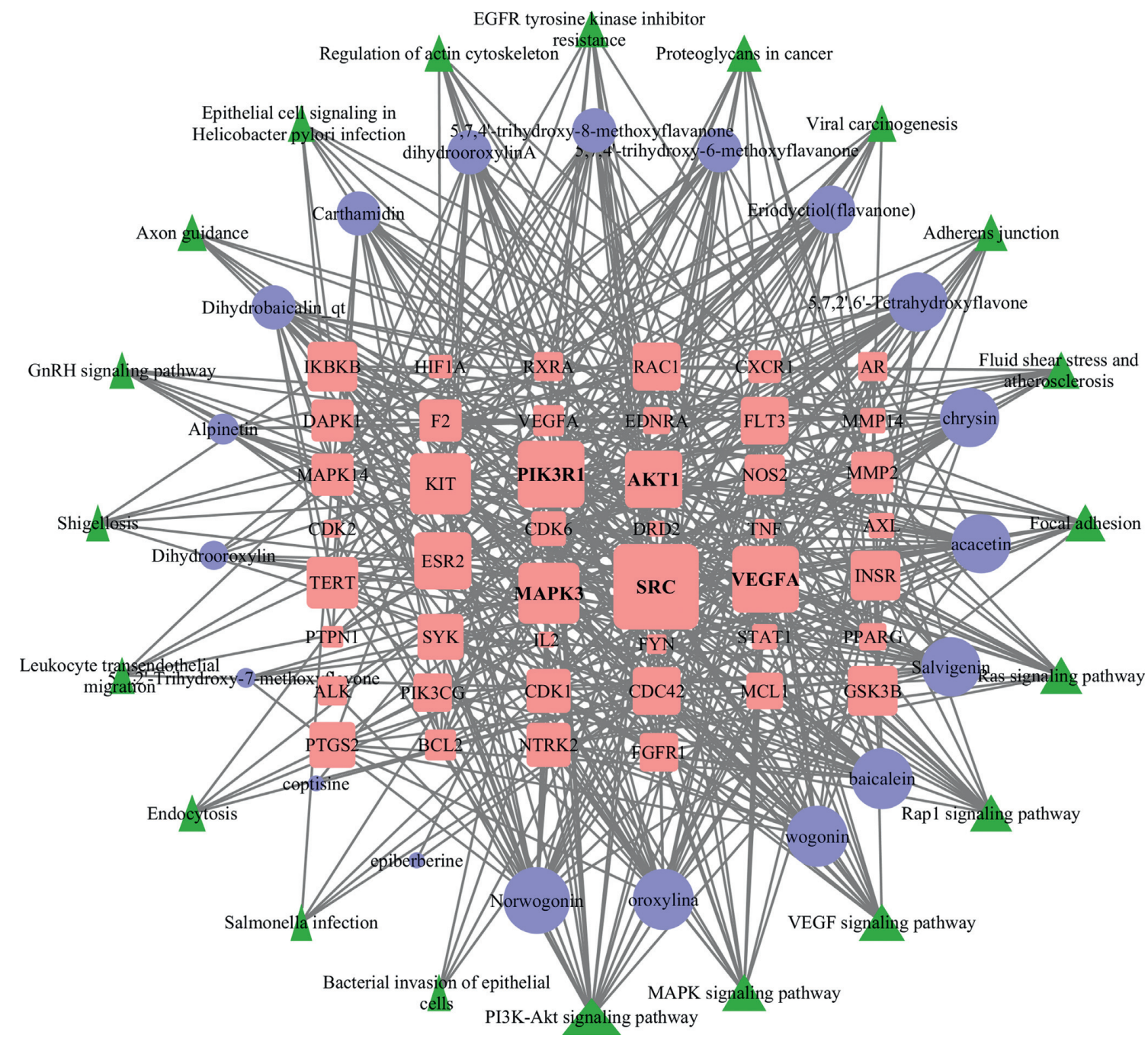

FIGURE 5: The network of "compound-target-pathway" of SBG for OSCC (the pink nodes represent the target of SBG for OSCC, the purple nodes represent the drug composition, and the green nodes represent the signaling pathway for SBG against OSCC).

TABLE 3: KEGG pathway enrichment results (top 20).

\begin{tabular}{|c|c|c|c|c|}
\hline Serial number & Pathway & Count & Gene ratio & $P$ value \\
\hline hsa04151 & PI3K-Akt signaling pathway & 25 & 0.291 & $1.63609 \mathrm{E}-08$ \\
\hline hsa04370 & VEGF signaling pathway & 18 & 0.209 & 7.83713E-07 \\
\hline hsa04010 & MAPK signaling pathway & 16 & 0.186 & $8.23084 \mathrm{E}-06$ \\
\hline hsa04014 & Ras signaling pathway & 12 & 0.140 & 8.40212E-06 \\
\hline hsa04015 & Rap1 signaling pathway & 11 & 0.128 & $1.49134 \mathrm{E}-05$ \\
\hline hsa04510 & Focal adhesion & 16 & 0.186 & $1.51741 \mathrm{E}-05$ \\
\hline hsa05418 & Fluid shear stress and atherosclerosis & 17 & 0.198 & $1.6281 \mathrm{E}-05$ \\
\hline hsa01521 & EGFR tyrosine kinase inhibitor resistance & 15 & 0.174 & $1.74787 \mathrm{E}-05$ \\
\hline hsa05205 & Proteoglycans in cancer & 13 & 0.151 & $2.97748 \mathrm{E}-05$ \\
\hline hsa04520 & Adherens junction & 11 & 0.128 & 3.22947E-05 \\
\hline hsa05203 & Viral carcinogenesis & 8 & 0.093 & 0.000800529 \\
\hline hsa04810 & Regulation of actin cytoskeleton & 9 & 0.105 & 0.003872469 \\
\hline hsa04360 & Axon guidance & 9 & 0.105 & 0.004695093 \\
\hline hsa05120 & Epithelial cell signaling in helicobacter pylori infection & 7 & 0.081 & 0.004967716 \\
\hline hsa04912 & GnRH signaling pathway & 9 & 0.105 & 0.006207927 \\
\hline hsa05131 & Shigellosis & 6 & 0.070 & 0.006899158 \\
\hline hsa04670 & Leukocyte transendothelial migration & 7 & 0.081 & 0.007712769 \\
\hline hsa05100 & Bacterial invasion of epithelial cells & 6 & 0.070 & 0.01033839 \\
\hline hsa04144 & Endocytosis & 8 & 0.093 & 0.019022329 \\
\hline hsa05132 & Salmonella infection & 5 & 0.058 & 0.032457397 \\
\hline
\end{tabular}




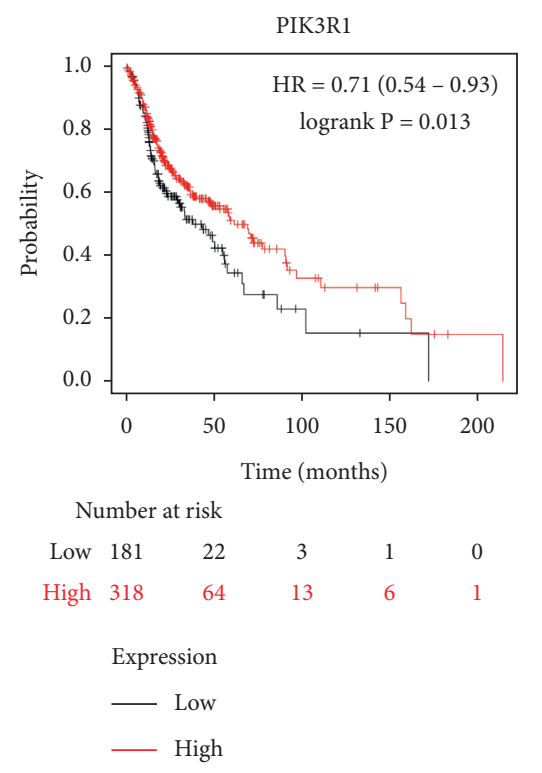

(a)

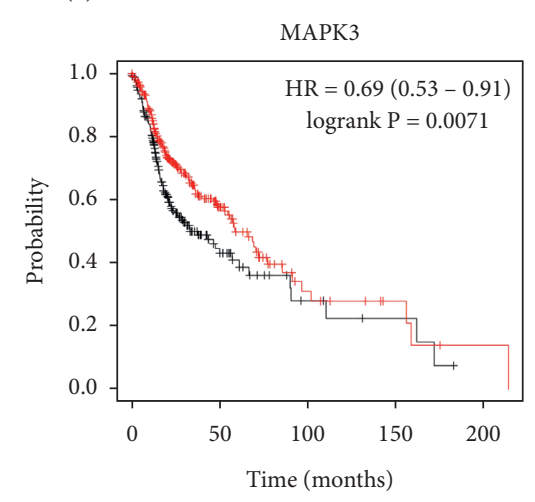

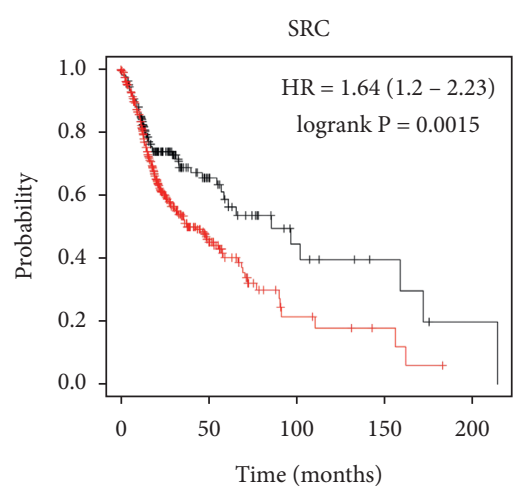
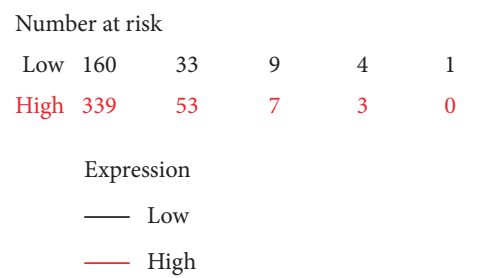

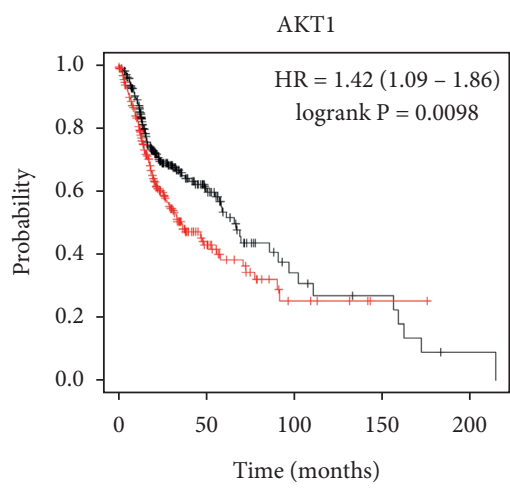

Number at risk

$\begin{array}{cccccc}\text { Low } & 260 & 48 & 10 & 6 & 1 \\ \text { High } & 239 & 38 & 6 & 1 & 0\end{array}$

Expression

- Low

— High

(b)

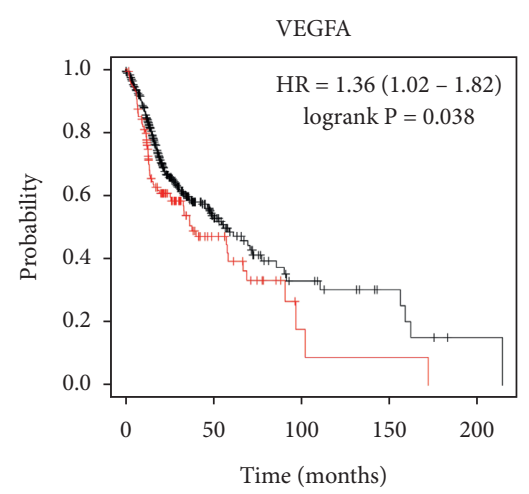

Number at risk

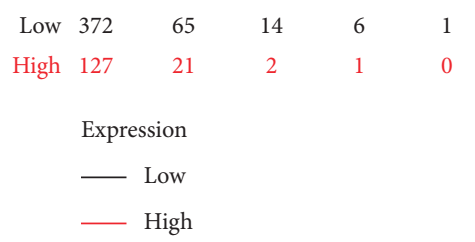

(e)

FIGURE 6: Survival curve analysis of five core target genes and median survival in OSCC patients. The five core target genes are (a) PIK3R1, (b) SRC, (c) AKT1, (d) MAPK3, and (e) VEGFA.

expression of MAPK3 is related to the invasion and metastasis of various tumor cells [46]. VEGFA is the most important proangiogenic factor and plays a crucial role in angiogenesis. Activated VEGFA can promote endothelial cell proliferation and migration and lead to new blood vessels' formation [47]. The above genes are associated with inflammation, cell proliferation, invasion, and angiogenesis, which are closely related to the occurrence and development of OSCC.

In our studies, KEGG enrichment analysis showed that 86 common targets were mainly related to the PI3K-Akt, VEGF, and MAPK signaling pathways. SBG may treat OSCC via the PI3K-Akt signaling pathway, which is an essential signaling pathway in regulating the cell cycle and can be activated by multiple types of stimulation or toxic injury. It plays a vital role in the chronic inflammatory response of OSCC [48]. As a downstream molecule of the P13K-Akt signal transduction pathway, mTOR plays a crucial role in tumorigenesis, invasion, metastasis, and angiogenesis. Phosphorylated mTOR (P-MTOR) overexpression is associated with poor prognosis in OSCC [49]. It was reported that $53 \%$ of tongue squamous cell carcinoma had p-MTOR overexpression [50]. The PI3K-Akt signaling pathway is altered in OSCC [51]. Invasion and migration can be suppressed in OSCC by inhibition of the PI3K-Akt signaling pathway [52]. Baicalein and wogonin induce apoptosis in 


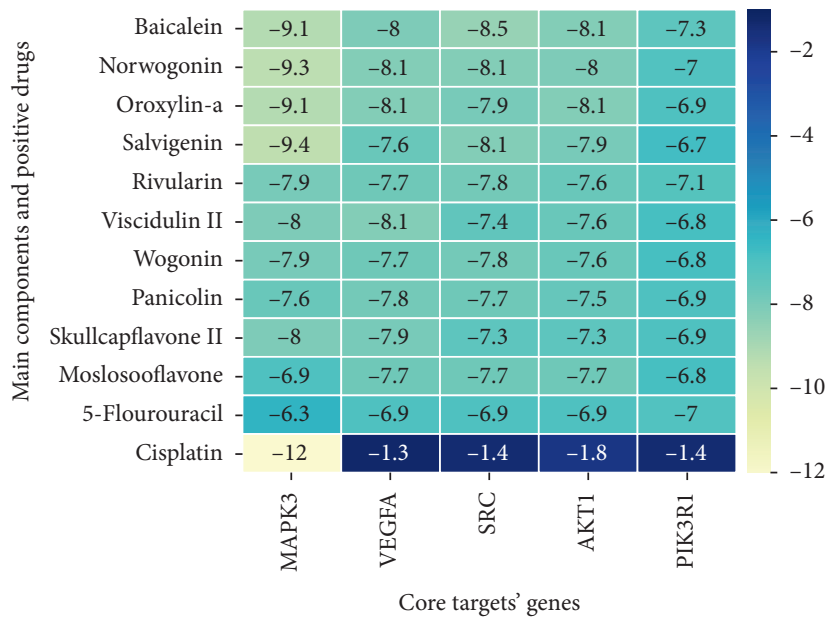

FIGURE 7: The binding energy of the main active components of SBG and core target genes.

TABLE 4: The binding energy of top 10 compounds of SBG with OSCC-related core targets.

\begin{tabular}{|c|c|c|c|c|c|c|c|}
\hline & \multirow{2}{*}{$\begin{array}{l}\text { Molecular } \\
\text { name }\end{array}$} & \multirow[b]{2}{*}{ CAS ID } & \multicolumn{5}{|c|}{ Core gene docking score $(\mathrm{kcal} / \mathrm{mol})$} \\
\hline & & & $\begin{array}{c}\text { MAPK3 } \\
\text { PDB ID (4QTB) }\end{array}$ & $\begin{array}{c}\text { VEGFA } \\
\text { PDB ID (3QTK) }\end{array}$ & $\begin{array}{c}\text { SRC } \\
\text { PDB ID (1FMK) }\end{array}$ & $\begin{array}{c}\text { AKT1 } \\
\text { PDB ID (4GV1) }\end{array}$ & $\begin{array}{c}\text { PIK3R1 } \\
\text { PDB ID (6D85) }\end{array}$ \\
\hline \multirow{10}{*}{ Core components } & Baicalein & $491-67-8$ & -9.1 & -8 & -8.5 & -8.1 & -7.3 \\
\hline & Norwogonin & $4443-09-8$ & -9.3 & -8.1 & -8.1 & -8 & -7 \\
\hline & Oroxylin-A & $480-11-5$ & -9.1 & -8.1 & -7.9 & -8.1 & -6.9 \\
\hline & Salvigenin & 19103-54-9 & -9.4 & -7.6 & -8.1 & -7.9 & -6.7 \\
\hline & Rivularin & $70028-59-0$ & -7.9 & -7.7 & -7.8 & -7.6 & -7.1 \\
\hline & Viscidulin II & $92519-93-2$ & -8.0 & -8.1 & -7.4 & -7.6 & -6.8 \\
\hline & Wogonin & $632-85-9$ & -7.9 & -7.7 & -7.8 & -7.6 & -6.8 \\
\hline & Panicolin & $41060-16-6$ & -7.6 & -7.8 & -7.7 & -7.5 & -6.9 \\
\hline & Skullcapflavone II & $55084-08-7$ & -8.0 & -7.9 & -7.3 & -7.3 & -6.9 \\
\hline & Moslosooflavone & $3570-62-5$ & -6.9 & -7.7 & -7.7 & -7.7 & -6.8 \\
\hline \multirow{2}{*}{ Positive drugs } & 5-Flourouracil & $51-21-8$ & -6.3 & -6.9 & -6.9 & -6.9 & \\
\hline & Cisplatin & $15663-27-1$ & -12.3 & -1.3 & -1.4 & -1.8 & -1.4 \\
\hline
\end{tabular}

many cancer cells by modulating the PI3K-Akt pathway $[53,54]$. Angiogenesis is an essential feature of the progression of OSCC and VEGF plays critical roles in OSCC tumor angiogenesis and metastasis [55]. Meanwhile, VEGFFlt-1 signaling can induce osteoclastogenesis in OSCC [56]. Previous studies have shown that the MAPK signaling pathway is involved in the development of OSCC, and TNF$\alpha$ regulates EMT through the MAPK signaling pathway to promote the invasion and metastasis of OSCC $[57,58]$. Baicalein plays an anti-inflammatory role by inhibiting the MAPK signaling pathway in pancreatitis, colon cancer, and thyroid cancer [59-61]. Thus, our data supported that SBG exerts its anti-OSCC effect by inhibiting cell proliferation, reducing inflammation via multiple pathways.

The GO of the BP was analyzed in our studies further to confirm the relationship between potential targets and OSCC. According to our analysis, the most enriched BP were positive regulation of transferase activity, cellular response to nitrogen compounds, and response to oxidative stress. Nitrogen acquisition and utilization have essential effects on cancer and immunity [62]. Oxidative stress and its induced oxidative damage are important factors in
OSCC formation and development $[63,64]$, which provided another insight into the mechanism of SBG in the treatment of OSCC.

In this study, the data of the core target genes on median survival of OSCC patients suggested that PIK3R1, SRC, AKT1, MAPK3, and VEGFA are potential therapeutic targets of SBG; there was a correlation with the survival time of OSCC patients. PIK3R1, SRC, AKT1, MAPK3, and VEGFA are common therapeutic targets for OSCC closely related to the survival and prognosis of OSCC patients $[31,53,65,66]$, which further supported the potential of SBG in the treatment of OSCC.

Molecular docking is the most common approach for evaluating component-target interactions. Baicalein, norwogonin, and oroxylin-A were the most stable active ingredient in core targets binding, and their docking scores were all $<-8.00 \mathrm{kcal} / \mathrm{mol}$. Wogonin, salvigenin, rivularin, skullcapflavone II, moslosooflavone, panicolin, and viscidulin showed a strong association with five core targets' docking scores being all<-7.00 kcal $/ \mathrm{mol}$. Nevertheless, compared to 5-fluorouracil and cisplatin, main components of SBG can bind to core targets of OSCC more stably on 


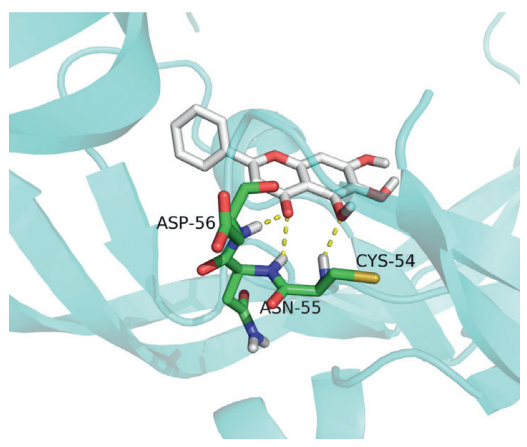

(a)

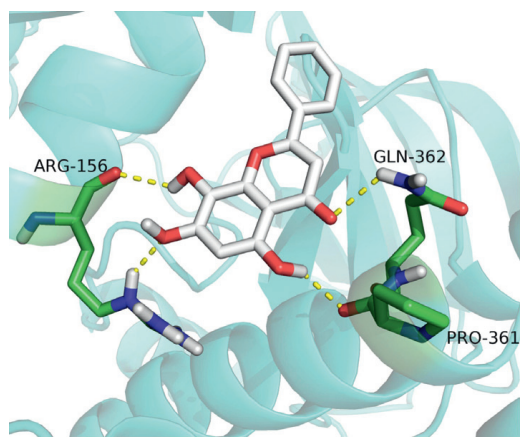

(d)

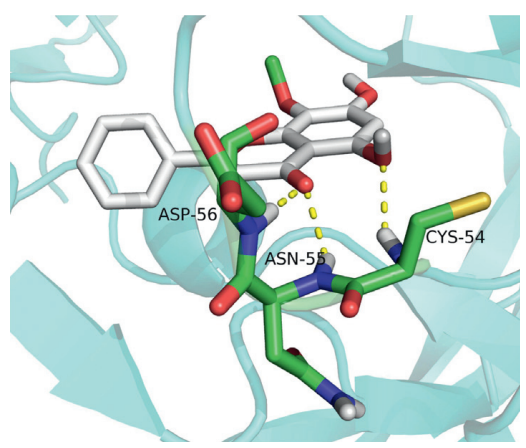

(b)

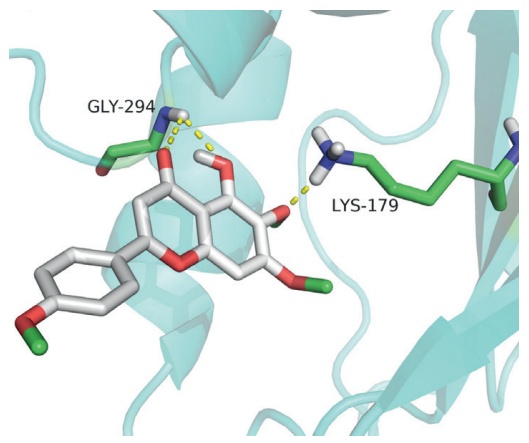

(e)

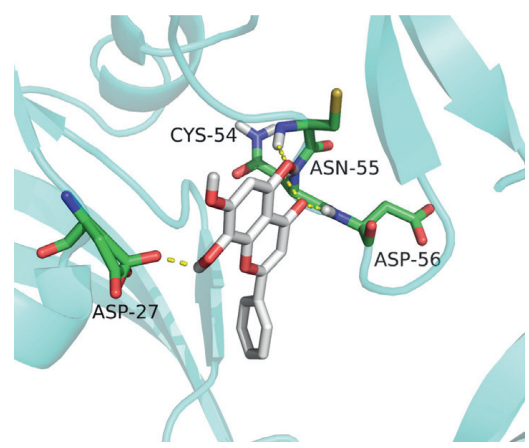

(c)

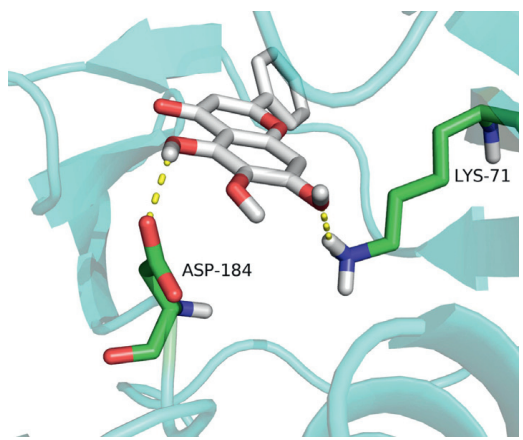

(f)

FIgURE 8: The result of molecular docking of the main components and core targets. (a) Baicalein-VEGFA. (b) Wogonin-VEGFA. (c) Norwogonin-VEGFA. (d) Norwogonin-SRC. (e) Salvigenin-AKT1. (f) Baicalein-MAPK3.

molecular docking anastomosis. These results suggest that an interaction does not mediate SBG treatment for OSCC between a single compound in SBG and a target protein in OSCC, but among multiple compounds in SBG and numerous target proteins in OSCC. Our data revealed that the effectiveness and mechanisms of SBG treatment on OSCC are complicated, which involved multicomponent interactions.

\section{Conclusions}

In the present study, network pharmacology identified 25 main active compounds in SBG and 86 genes targeted by OSCC. Five core targets (PIK3R1, SRC, AKT1, MAPK3, and VEGFA) from complex networks through the integration of network pharmacology were predicted to be critical targets of SBG in treating OSCC. Besides, we found that the PI3KAkt signaling pathway may occupy core status in the antiOSCC system. Results from molecular docking further revealed that several SBG compounds exhibited an excellent affinity for their core targets. These results elucidate the potentially effective mechanisms of SBG to treat OSCC on multiple targets and multiple pathways, which also shed a light on the development of the new drug for OSCC.

\section{Abbreviations}

OSCC: Oral squamous cell carcinoma

SBG: $\quad$ Scutellaria baicalensis Georgi
TCMSP: Traditional Chinese Medicine Systems Pharmacology Database

OMIM: Online Mendelian Inheritance in Man

TTD: $\quad$ Therapeutic Target Database

PPI: $\quad$ Protein-protein interaction

GO: $\quad$ Gene Ontology

KEGG: Kyoto Encyclopedia of Genes and Genomes

CC: $\quad$ Cellular components

MF: Molecular functions

BP: Biological processes

TCM: Traditional Chinese medicine

ADME: Absorption, distribution, metabolism, and excretion

OB: $\quad$ Oral bioavailability

DL: Drug-likeness

STRING: Search Tool for the Retrieval of Interacting Genes/Proteins

TCGA: The Cancer Genome Atlas

GEO: $\quad$ Gene Expression Omnibus

PIK3R1: Phosphoinositide-3-kinase regulatory subunit 1

SRC: $\quad$ SRC proto-oncogene

AKT1: $\quad$ AKT serine/threonine kinase 1

MAPK3: Mitogen-activated protein kinase 3

VEGFA: Vascular endothelial growth factor A.

\section{Data Availability}

All the data are available from the first author upon reasonable request. The data used to support the findings of this 
study are included within the supplementary information files.

\section{Disclosure}

Fanfan Hou and Yang Liu are considered as joint first authors.

\section{Conflicts of Interest}

The authors declare that there are no conflicts of interest.

\section{Authors' Contributions}

Fanfan Hou and Yang Liu contributed equally to this work, contributed to the conceptualization, and conducted the network analysis, data interpretation, and manuscript preparation. Fang Zhang and YaHsin Cheng contributed to the direction and concept of the experiment, conducted data interpretation, and discussed the data. Ni Zhang contributed to the data interpretation and molecular docking simulation. Wenpeng Yan contributed to the data collection, literature search, and funds' collection. All authors read and agreed to the published version of the manuscript.

\section{Acknowledgments}

This work was supported by the Youth Science and Technology Research Fund of Shanxi Province (no. 201801D221270).

\section{Supplementary Materials}

Table S1: detailed information of active compounds in SBG. Table S2: target gene-related active compounds of SBG. Table S3: list of OSCC-related genes in the GeneCards database, OMIM, and TTD. Table S4: the putative targets of SBG against OSCC. Table S5: topological analysis of the PPI network. Table S6: topological analysis of the compoundtarget-disease network. Table S7: the GO enrichment analysis for intersection targets between compound and OSCC-related targets. Table S8: the enriched KEGG pathways for intersection targets between compound and ADrelated targets. Table S9: the results of molecular docking. (Supplementary Materials)

\section{References}

[1] F. Bray, J. Ferlay, I. Soerjomataram, R. L. Siegel, L. A. Torre, and A. Jemal, "Global cancer statistics 2018: GLOBOCAN estimates of incidence and mortality worldwide for 36 cancers in 185 countries," CA: A Cancer Journal for Clinicians, vol. 68, no. 6, pp. 394-424, 2018.

[2] M. Kumar, R. Nanavati, T. Modi, and C. Dobariya, "Oral cancer: etiology and risk factors: a review," Journal of Cancer Research and Therapeutics, vol. 12, no. 2, pp. 458-463, 2016.

[3] Y. Zhang, Y. Yuan, H. Wu et al., "Effect of verbascoside on apoptosis and metastasis in human oral squamous cell carcinoma," International Journal of Cancer, vol. 143, no. 4, pp. 980-991, 2018.
[4] S. Furness, A. M. Glenny, H. V. Worthington et al., "Interventions for the treatment of oral cavity and oropharyngeal cancer: chemotherapy," Cochrane Database of Systematic Reviews, vol. 4, Article ID Cd006386, 2011.

[5] Q. Zhao, X.-Y. Chen, and C. Martin, "Scutellaria baicalensis, the golden herb from the garden of Chinese medicinal plants," Science Bulletin, vol. 61, no. 18, pp. 1391-1398, 2016.

[6] Z.-L. Wang, S. Wang, Y. Kuang, Z.-M. Hu, X. Qiao, and M. Ye, "A comprehensive review on phytochemistry, pharmacology, and flavonoid biosynthesis of Scutellaria baicalensis," Pharmaceutical Biology, vol. 56, no. 1, pp. 465-484, 2018.

[7] T. Zhao, H. Tang, L. Xie et al., "Scutellaria baicalensis Georgi. (Lamiaceae): a review of its traditional uses, botany, phytochemistry, pharmacology and toxicology," Journal of Pharmacy and Pharmacology, vol. 71, no. 9, pp. 1353-1369, 2019.

[8] Z. Gao, Y. Zhang, H. Zhou, and J. Lv, "Baicalein inhibits the growth of oral squamous cell carcinoma cells by downregulating the expression of transcription factor Sp1," International Journal of Oncology, vol. 56, no. 1, pp. 273-282, 2020.

[9] D. Sato, S. Kondo, K. Yazawa et al., "The potential anticancer activity of extracts derived from the roots of Scutellaria baicalensis on human oral squamous cell carcinoma cells," Molecular and Clinical Oncology, vol. 1, no. 1, pp. 105-111, 2013.

[10] D. Y. Zhang, J. Wu, F. Ye et al., "Inhibition of cancer cell proliferation and prostaglandin E2 synthesis by Scutellaria baicalensis," Cancer Research, vol. 63, no. 14, pp. 4037-4043, 2003.

[11] S.-H. Liu, W.-C. Chuang, W. Lam, Z. Jiang, and Y.-C. Cheng, "Safety surveillance of traditional Chinese medicine: current and future," Drug Safety, vol. 38, no. 2, pp. 117-128, 2015.

[12] G. Yu, Z. Luo, Y. Zhou et al., "Uncovering the pharmacological mechanism of Carthamus tinctorius L. on cardiovascular disease by a systems pharmacology approach," Biomedicine and Pharmacotherapy, vol. 117, Article ID 109094, 2019.

[13] L. Pinzi and G. Rastelli, "Molecular docking: shifting paradigms in drug discovery," International Journal of Molecular Sciences, vol. 20, no. 18, 2019.

[14] B. Győrffy, P. Surowiak, J. Budczies, and A. Lánczky, "Online survival analysis software to assess the prognostic value of biomarkers using transcriptomic data in non-small-cell lung cancer," PLoS One, vol. 8, no. 12, Article ID e82241, 2013.

[15] J. Ru, P. Li, J. Wang et al., "TCMSP: a database of systems pharmacology for drug discovery from herbal medicines," Journal of Cheminformatics, vol. 6, no. 13, 2014.

[16] X. X. Xu, J. P. Bi, L. Ping, P. Li, and F. Li, "A network pharmacology approach to determine the synergetic mechanisms of herb couple for treating rheumatic arthritis," Drug Design, Development and Therapy, vol. 12, pp. 967-979, 2018.

[17] Y. Wang, S. H. Bryant, T. Cheng et al., "PubChem BioAssay: 2017 update," Nucleic Acids Research, vol. 45, no. D1, pp. D955-d963, 2017.

[18] A. Daina, O. Michielin, and V. Zoete, "SwissTargetPrediction: updated data and new features for efficient prediction of protein targets of small molecules," Nucleic Acids Research, vol. 47, no. W1, pp. W357-W364, 2019.

[19] G. Stelzer, N. Rosen, I. Plaschkes et al., "The GeneCards suite: from gene data mining to disease Genome sequence analyses," Current Protocols in Bioinformatics, vol. 54, 2016.

[20] J. S. Amberger, C. A. Bocchini, F. Schiettecatte, A. F. Scott, and A. Hamosh, "OMIM.org: online Mendelian Inheritance in 
Man (OMIM), an online catalog of human genes and genetic disorders," Nucleic Acids Research, vol. 43, no. D1, pp. D789-D798, 2015.

[21] X. Chen, Z. L. Ji, and Y. Z. Chen, "TTD: therapeutic target database," Nucleic Acids Research, vol. 30, no. 1, pp. 412-415, 2002.

[22] L. Sun, S. Dong, Y. Ge et al., "DiVenn: an interactive and integrated web-based visualization tool for comparing gene lists," Frontiers in Genetics, vol. 10, no. 10, pp. 421-426, 2019.

[23] P. Shannon, A. Markiel, O. Ozier et al., "Cytoscape: a software environment for integrated models of biomolecular interaction networks," Genome Research, vol. 13, no. 11, pp. 2498-2504, 2003.

[24] D. Szklarczyk, J. H. Morris, H. Cook et al., "The STRING database in 2017: quality-controlled protein-protein association networks, made broadly accessible," Nucleic Acids Research, vol. 45, no. D1, pp. D362-D368, 2017.

[25] Y. Zhou, B. Zhou, L. Pache et al., "Metascape provides a biologist-oriented resource for the analysis of systems-level datasets," Nature Communications, vol. 10, no. 1, Article ID $1523,2019$.

[26] N. T. Doncheva, J. H. Morris, J. Gorodkin, and L. J. Jensen, "Cytoscape StringApp: network analysis and visualization of proteomics data," Journal of Proteome Research, vol. 18, no. 2, pp. 623-632, 2019.

[27] A. Colaprico, T. C. Silva, C. Olsen et al., "TCGAbiolinks: an R/ Bioconductor package for integrative analysis of TCGA data," Nucleic Acids Research, vol. 44, no. 8, p. e71, 2016.

[28] T. Gaillard, "Evaluation of AutoDock and AutoDock vina on the CASF-2013 benchmark," Journal of Chemical Information and Modeling, vol. 58, no. 8, pp. 1697-1706, 2018.

[29] A. Aupérin, "Epidemiology of head and neck cancers: an update," Current Opinion in Oncology, vol. 32, no. 3, pp. 178-186, 2020.

[30] Y. Xiang, Z. Guo, P. Zhu, J. Chen, and Y. Huang, "Traditional Chinese medicine as a cancer treatment: modern perspectives of ancient but advanced science," Cancer Medicine, vol. 8, no. 5, pp. 1958-1975, 2019.

[31] L. Wang, W. Chen, M. Li, F. Zhang, K. Chen, and W. Chen, “A review of the ethnopharmacology, phytochemistry, pharmacology, and quality control of Scutellaria barbata D. Don," Journal of Ethnopharmacology, vol. 254, Article ID 112260, 2020.

[32] F. Perez-Vizcaino and C. G. Fraga, "Research trends in flavonoids and health," Archives of Biochemistry and Biophysics, vol. 646, pp. 107-112, 2018.

[33] C. Yu, J. Zeng, Z. Yan, Z. Ma, S. Liu, and Z. Huang, "Baicalein antagonizes acute megakaryoblastic leukemia in vitro and in vivo by inducing cell cycle arrest," Cell and Bioscience, vol. 6, p. 20, 2016.

[34] M. Li-Weber, "New therapeutic aspects of flavones: the anticancer properties of Scutellaria and its main active constituents Wogonin, Baicalein and Baicalin," Cancer Treatment Reviews, vol. 35, no. 1, pp. 57-68, 2009.

[35] M. Li-Weber, "Targeting apoptosis pathways in cancer by Chinese medicine," Cancer Letters, vol. 332, no. 2, pp. 304-312, 2013.

[36] L. Lu, Q. Guo, and L. Zhao, "Overview of oroxylin A: a promising flavonoid compound," Phytotherapy Research, vol. 30, no. 11, pp. 1765-1774, 2016.

[37] S. Noori, Z. M. Hassan, B. Yaghmaei, and M. Dolatkhah, "Antitumor and immunomodulatory effects of salvigenin on tumor bearing mice," Cellular Immunology, vol. 286, no. 1-2, pp. 16-21, 2013.
[38] N. Namazi Sarvestani, H. Sepehri, L. Delphi, and M. M. Farimani, "Eupatorin and salvigenin potentiate doxorubicin-induced apoptosis and cell cycle arrest in HT-29 and SW948 human colon cancer cells," Asian Pacific Journal of Cancer Prevention, vol. 19, no. 1, pp. 131-139, 2018.

[39] P. L. B. Domingos, M. G. Souza, T. A. Guimarães et al., "Hypoxia reduces the E-cadherin expression and increases OSCC cell migration regardless of the E-cadherin methylation profile," Pathology-Research and Practice, vol. 213, no. 5, pp. 496-501, 2017

[40] J. P. Joseph, M. K. Harishankar, A. A. Pillai, and A. Devi, "Hypoxia induced EMT: a review on the mechanism of tumor progression and metastasis in OSCC," Oral Oncology, vol. 80, pp. 23-32, 2018.

[41] L. Jing, R. Gao, J. Zhang et al., "Norwogonin attenuates hypoxia-induced oxidative stress and apoptosis in PC12 cells," BMC Complementary Medicine and Therapies, vol. 21, no. 1, p. 18, 2021.

[42] J. Vallejo-Díaz, M. Chagoyen, M. Olazabal-Morán, A. González-García, and A. C. Carrera, "The opposing roles of PIK3R1/p85 $\alpha$ and PIK3R2/p85 $\beta$ in cancer," Trends in Cancer, vol. 5, no. 4, pp. 233-244, 2019.

[43] J. X. Wang, X. J. Jia, Y. Liu et al., "Silencing of miR-17-5p suppresses cell proliferation and promotes cell apoptosis by directly targeting PIK3R1 in laryngeal squamous cell carcinoma," Cancer Cell International, vol. 20, no. 14, 2020.

[44] A. Sirvent, C. Benistant, and S. Roche, "Oncogenic signaling by tyrosine kinases of the SRC family in advanced colorectal cancer," American Journal of Cancer Research, vol. 2, no. 4, pp. 357-371, 2012.

[45] K.-I. Nakashiro, H. Tanaka, H. Goda et al., "Identification of Akt1 as a potent therapeutic target for oral squamous cell carcinoma," International Journal of Oncology, vol. 47, no. 4, pp. 1273-1281, 2015.

[46] H. Y. Cao, C. H. Xiao, H. J. Lu et al., "MiR-129 reduces CDDP resistance in gastric cancer cells by inhibiting MAPK3," European Review for Medical and Pharmacological Sciences, vol. 23, no. 15, pp. 6478-6485, 2019.

[47] D. Tang, H. Nagano, H. Yamamoto et al., "Angiogenesis in cholangiocellular carcinoma: expression of vascular endothelial growth factor, angiopoietin-1/2, thrombospondin-1 and clinicopathological significance," Oncology Reports, vol. 15 , no. 3, pp. 525-532, 2006.

[48] P. Velu, A. Vijayalakshmi, and V. Vinothkumar, "Inhibiting the PI3K/Akt, NF- $\kappa \mathrm{B}$ signalling pathways with syringic acid for attenuating the development of oral squamous cell carcinoma cells SCC131," Journal of Pharmacy and Pharmacology, vol. 72, 2020.

[49] J. Patel, S. A. Nguyen, B. Ogretmen, J. S. Gutkind, C. A. Nathan, and T. Day, "mTOR inhibitor use in head and neck squamous cell carcinoma: a meta-analysis on survival, tumor response, and toxicity," Laryngoscope Investigative Otolaryngology, vol. 5, no. 2, pp. 243-255, 2020.

[50] S.-H. Li, C.-Y. Chien, W.-T. Huang et al., "Prognostic significance and function of mammalian target of rapamycin in tongue squamous cell carcinoma," Scientific Reports, vol. 7, no. 1, Article ID 8178, 2017.

[51] S. M. Rothenberg and L. W. Ellisen, "The molecular pathogenesis of head and neck squamous cell carcinoma," Journal of Clinical Investigation, vol. 122, no. 6, pp. 1951-1957, 2012.

[52] Y. Du, J. Zhang, Y. Meng, M. Huang, W. Yan, and Z. Wu, "MicroRNA-143 targets MAPK3 to regulate the proliferation and bone metastasis of human breast cancer cells," $A M B$ Express, vol. 10, no. 1, p. 134, 2020. 
[53] M. Yu, B. Qi, W. Xiaoxiang, J. Xu, and X. Liu, "Baicalein increases cisplatin sensitivity of A549 lung adenocarcinoma cells via PI3K/Akt/NF- $\kappa \mathrm{B}$ pathway," Biomedicine and Pharmacotherapy, vol. 90, pp. 677-685, 2017.

[54] C. Hu, M. Xu, R. Qin, W. Chen, and X. Xu, "Wogonin induces apoptosis and endoplasmic reticulum stress in HL-60 leukemia cells through inhibition of the PI3K-AKT signaling pathway," Oncology Reports, vol. 33, no. 6, pp. 3146-3154, 2015.

[55] M.-Y. Lien, A.-C. Chang, H.-C. Tsai et al., "Monocyte chemoattractant protein 1 promotes VEGF-A expression in OSCC by activating ILK and MEK1/2 signaling and downregulating miR-29c," Frontiers in Oncology, vol. 10, Article ID 592415, 2020.

[56] A. Subarnbhesaj, M. Miyauchi, C. Chanbora et al., "Roles of VEGF-Flt-1 signaling in malignant behaviors of oral squamous cell carcinoma," PLoS One, vol. 12, no. 11, Article ID e0187092, 2017.

[57] X.-W. Zhao, J.-P. Zhou, Y.-L. Bi et al., "The role of MAPK signaling pathway in formation of EMT in oral squamous carcinoma cells induced by TNF- $\alpha$," Molecular Biology Reports, vol. 46, no. 3, pp. 3149-3156, 2019.

[58] T. Jin, Y. Guo, Z. Huang et al., "Vitamin D inhibits the proliferation of Oral Squamous Cell Carcinoma by suppressing lncRNA LUCAT1 through the MAPK pathway," Journal of Cancer, vol. 11, no. 20, pp. 5971-5981, 2020.

[59] W.-1. Pu, R.-y. Bai, K. Zhou et al., "Baicalein attenuates pancreatic inflammatory injury through regulating MAPK, STAT 3 and NF- $\kappa$ B activation," International Immunopharmacology, vol. 72, pp. 204-210, 2019.

[60] M. Q. Su, Y. R. Zhou, X. Rao et al., "Baicalein induces the apoptosis of HCT116 human colon cancer cells via the upregulation of DEPP/Gadd45a and activation of MAPKs," International Journal of Oncology, vol. 53, no. 2, pp. 750-760, 2018.

[61] S. E. Han, C. H. Park, I. S. Nam-Goong, Y. I. Kim, and E. S. Kim, "Anticancer effects of baicalein in FRO thyroid cancer cells through the up-regulation of ERK/p38 MAPK and akt pathway," In Vivo, vol. 33, no. 2, pp. 375-382, 2019.

[62] K. Kurmi and M. C. Haigis, "Nitrogen metabolism in cancer and immunity," Trends in Cell Biology, vol. 30, no. 5, pp. 408-424, 2020.

[63] P. M. Biselli-Chicote, A. T. Lotierzo, J. M. Biselli et al., "Atorvastatin increases oxidative stress and inhibits cell migration of oral squamous cell carcinoma in vitro," Oral Oncol, vol. 90, no. 3, pp. 109-114, 2019.

[64] T. N. Hsu, C. M. Huang, C. S. Huang et al., "Targeting FAT1 inhibits carcinogenesis, induces oxidative stress and enhances cisplatin sensitivity through deregulation of LRP5/WNT2/ GSS signaling Axis in oral squamous cell carcinoma," Cancers, vol. 11 , no. $12,2019$.

[65] F. Hermida-Prado, R. Granda-Díaz, N. Del-Río-Ibisate et al., "The differential impact of SRC expression on the prognosis of patients with head and neck squamous cell carcinoma," Cancers (Basel), vol. 11, no. 11, 2019.

[66] G. T. Peterle, L. L. Maia, L. O. Trivilin et al., "PAI-1, CAIX, and VEGFA expressions as prognosis markers in oral squamous cell carcinoma," Journal of Oral Pathology and Medicine, vol. 47, no. 6, pp. 566-574, 2018. 